\title{
Antibiotic Consumption in Hospitalized Children at the University Clinic for Pediatric Diseases - Skopje
}

\author{
Iskra Pechijareva-Sadikarijo ${ }^{1 *}$, Zorica Naumovska ${ }^{2}$, Ognen Petrovski ${ }^{3}$, Katerina Stavrikj ${ }^{4}$, Trajan Balkanov $^{3}$ \\ ${ }^{1}$ Macedonian Agency for Medicines and Medical Devices, Skopje, Republic of Macedonia; ${ }^{2}$ Institute for Pharmaceutical \\ Chemistry, Faculty of Pharmacy, Skopje, Republic of Macedonia; ${ }^{3}$ Institute for Clinical Pharmacology and Toxicology, Medical \\ Faculty, Skopje, Republic of Macedonia; ${ }^{4}$ University Clinic for Pediatric Diseases, Skopje, Republic of Macedonia
}

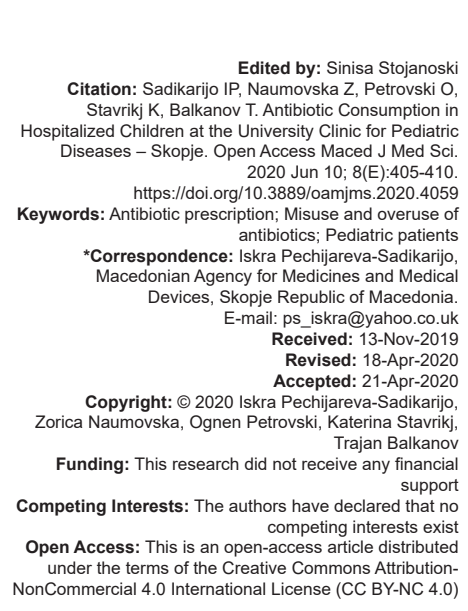

Introduction

The misuse and overuse of antibiotics contribute to the development of antimicrobial resistance. Incidence of serious infections caused by multidrug-resistant microorganisms has increased; multidrug-resistance microorganisms continue to pose a serious threat for the public health as they do not respond to conventional treatment. It is estimated that in the EU countries two million people were hospitalized due to infectious diseases, and about two hundred thousand died annually [1]. According to the official data for EU/EEA in 2017, the average consumption of antibiotics for systemic use (outside of hospitals) was 21.8 defined daily dose/1000 inhabitants/ day (DDD/TID) (10.1 in Nederland to 33.6 in Cyprus). In the hospital sector, official data suggested that variations are much smaller with the lowest consumption is in the Netherlands, with 1 DDD/1000 inhabitant per day to 2.8 DDD in Finland. On average, hospitals in the EU spend 2 DDD/1000 inhabitants. Still over $90 \%$ of the consumption of antimicrobial drugs is outside of hospitals. Most frequently prescribed were penicillin's in combination with $\beta$-lactamase inhibitors, broad-spectrum penicillin's, and cephalosporins followed by macrolides and tetracyclines [2]. Significant differences in prescribing habits were identified among the EU countries for the type and quantity of antibacterial drugs. Evident differences were identified for the prescription of beta-lactamase sensitive penicillin's (natural penicillin and aminopenicillins) within the EU. Furthermore, the consumption ratio of broadspectrum penicillin's, cephalosporins, and macrolides with that of narrow-spectrum penicillin's, cephalosporins, and macrolides in the EU/EEA was 2.3 [3].

In the Republic of North Macedonia, according to the Health Insurance Fund (HIF) data, in the period 2012-2018, the consumption of prescription antimicrobials in primary health care ranged from 17 to about 20 DDD per 1000 insured persons. These data do not cover antibiotics purchased by private insurers or patients on their own.

The data obtained by Health Insurance Fund (HIF) of the Republic of North Macedonia, the share of beta-lactamase-sensitive penicillin's is $14.5 \%$ of the total prescribed antimicrobial drugs in the period 2012-2018. The ratio of broad-spectrum versus narrow-spectrum penicillins is 2 . In the second place 
of the prescribed, antibiotics are cephalosporins with an average of about 456000 prescriptions (2.22\%), fluoroquinolones 265000 (1.24\%), and macrolides with an average of 244000 (1.19\%) prescriptions/ year. Tetracyclines, which are in the third place in the EU, are in the last place in the Republic of North Macedonia, after the prescriptions for cotrimoxazole $0.3 \%$ (60 000) and lincosamides $0.16 \%$ (32.000), with about 3000 (0.014\%) prescriptions for doxycycline [4].

The average number of prescriptions for antibacterial drugs prescribed annually was about 1.9 million in the evaluated period. According to the most optimistic estimates, the total consumption of antibiotics in the Republic of North Macedonia would be around 30 DDD per 1000 inhabitants, and together with hospital medicines, it is assumed to be around 32 DDD per 1000 inhabitants. The most pessimistic estimates are that the total consumption of antimicrobial drugs in the Republic of North Macedonia is up to 40 DDD per 1000 inhabitants (4 of 100 inhabitants use antibiotics every day). Almost one-third of the prescribed drugs are amoxicillinclavulanic acid and the number of prescriptions exceeded 500,000 in 2014 or over 5 DDD/1000 inhabitants. Having in consideration the DDDs of antimicrobial drugs that the patients are buying in pharmacies outside health insurance, it could be approximated that up to 10 DDDs/1000 inhabitants are consumed, which would be almost the same as the total consumption of all systemic antibacterial drugs used in the Netherlands (11 DDDs ambulatory primary care and 1DDD in-hospital) [3].

Monitoring the prescription of antibiotics is of crucial importance, due to more distinct bacterial resistance, the occurrence of adverse reactions and treatment costs. A particular challenge is monitoring the usage ofantibioticsand the resistance ofbacteriainhospital settings with a pediatric population [5], [6], [7], [8], [9]

Bacteria isolated in biological samples from children under the age of two shows a higher percentage of antibiotic resistance compared to bacteria isolated in older children while isolates obtained from the hospitals are more resistant in comparison to the isolates from primary care [10], [11], [12], [13].

The Anatomical Therapeutic Chemical Classification/Defined Daily Dose (ATC/DDD) system is the standard method for measuring the consumption of drugs that allow analyses and comparing consumption between different countries, regions, and health-care institutions [14], [15]. Drug consumption in hospitals is expressed with the DDD/100BD statistical unit, where the used antibiotics expressed in daily defined dosesDDD are correlated with the number of hospitals spent days-BD (bed/day) in a given period of time.

The study monitors the consumption of systemic antibiotics that are classified according to the ATC classification as J01 - antibiotics for systemic use. DDD is a statistical unit for monitoring the usage of medical products that expresses the average daily dose of the specified drug for the most common indication for which it is used.

There are several reasons for different levels of resistance that includes antibiotic use, more difficult diseases to diagnose, quality of hospital care, immunization rate, and social factors in the country. It is not always possible to determine the ratio of resistant infections caused by each individual factor. Data from the European Antimicrobial Resistance Data Surveillance System show that countries in northern Europe, such as the Scandinavian countries and the Netherlands, have a low rate of resistance, and in countries, in the southern parts of Europe, the rate is higher. Countries with a low rate of resistance to antibiotics have generally lower use of antimicrobials and, by analogy, countries with a higher degree of resistance use more antibiotics [3]. The data on the antibiotic consumption and bacterial resistance in the hospitalized pediatric population in the Republic of North Macedonia are limited.

The conducted study aims to evaluate and compare the antibiotics consumption in the pediatric hospitalized population at the University Clinic for Pediatric Diseases in Skopje for a period of 3 months in the year 2018 and 2019 based on hospital departments' data.

\section{Materials and Methods}

The observational pharmacoepidemiological study was conducted at the University Clinic for Pediatric Diseases in Skopje. The data for antibiotics consumption were obtained from the hospital pharmacy, in the study, we have compared the antibiotics consumption between the Quarter 1 (January, February, and March) of 2018 and Quarter 1 (January, February, and March) of 2019.

The University Clinic for Pediatric Diseases in Skopje is tertiary health-care institution with 177 hospital beds, divided in the following twelve departments: Hematology, oncology, pulmonology, endocrinology and genetics, immunology, neonatology, gastroenterohepatology, infant, metabolism and cystic fibrosis, cardiology with rheumatology, nephrology, neurology, and intensive care. The number of hospitalized patients in Quarter 1 (January, February, and March) of 2018 and Quarter 1 (January, February, and March) of 2019 is presented in Table 1.

Consumption of systemic antibiotics was evaluated based on dispensed systemic antibiotics J01 (regardless of the indication for which they were used - prophylaxis or therapy) for each department provided from the hospital pharmacy information system (assessed by type and volume, departments, ATC classification, and generic drug name). Total spent hospital days (bed/day) BD- i.e. the number of hospitalizations per day during a certain period of 
Table 1: Departments and number of hospitalized patients

\begin{tabular}{llll}
\hline Departments & No of beds & $\begin{array}{l}\text { From 1.01. } \\
\text { to31.3.2018 }\end{array}$ & $\begin{array}{l}\text { From 1.01. } \\
\text { to31.3.2019 }\end{array}$ \\
\hline Intensive care & 18 & 49 & 42 \\
Oncology & 14 & 53 & 49 \\
Hematology & 12 & 116 & 129 \\
Cardiology with rheumatology & 14 & 128 & 136 \\
Endocrinology and genetics & 12 & 178 & 339 \\
Pulmonology A & 14 & 185 & 109 \\
Pulmonology B & 12 & 103 & 116 \\
Immunology & 14 & 151 & 134 \\
Gastroenterohepatology & 12 & 118 & 141 \\
Infant, metabolism, and cystic fibrosis & 14 & 135 & 119 \\
Neurology & 12 & 146 & 137 \\
Neonatology & 17 & 111 & 96 \\
Nephrology & 12 & 135 & 90 \\
Total & $\mathbf{1 7 7}$ & $\mathbf{1 6 0 8}$ & $\mathbf{1 6 3 7}$ \\
\hline & & & \\
\hline
\end{tabular}

time and the index of occupancy of hospital beds were collected on a monthly basis from the Clinic.

The quantity of antimicrobial drugs was converted into a number of defined daily doses (DDD/100 bed-days (DBD) through the anatomical-therapeuticchemical (ATC) and DDD drug classification [16].

Antibiotics consumption in the hospital (DDD/100BD) was calculated with the following formula:

$\begin{aligned} \mathrm{DDD} / 100 \mathrm{BD} & =\frac{\text { Number of spent units antibiotic }(\mathrm{mg})}{\mathrm{DDD}(\mathrm{mg}) \times \text { No. of days } \times \text { No. of beds }} \\ & \times \frac{100 \text { beds }}{\text { Index of occupied beds }}\end{aligned}$

\section{Results}

The total amount of utilized antibacterial drugs with ATC code J01 for the first quarter (3 months January, February, and March 2018) at the University Clinic for Pediatric Diseases in Skopje was 33.65 DDD/100BD in comparison to the first quarter of 2019 when it declined to $32.09 \mathrm{DDD} / 100 \mathrm{BD}$. The evaluation of antibiotics consumption in the first Quarter of 2018 versus 2019 was performed after the implementation of restriction policy and control of the prescription of certain antibiotics. The results are presented in Table 2.

As presented in Table 2, the most frequently utilized antibiotics were the group of cephalosporins, in both Q1-2018 and Q1-2019. There was an evident reduction of consumption of the first and second generation of cephalosporins (cefadroxil 0.28 DDD/100BD in 2018 versus $0.16 \mathrm{DDD} / 100 \mathrm{BD}$ in 2019 and twofold declination of consumption of the oral form of cefaclor from 0.18 DDD/100BD in 2018 to $0.09 \mathrm{DDD} / 100 \mathrm{BD}$ in 2019). The evident increase was evaluated in the use of $3^{\text {rd }}$ and $4^{\text {th }}$ generation of cephalosporins (cefotaxime from $0.18 \mathrm{DDD} / 100 \mathrm{BD}$ to 2.15 DDD/100BD ceftriaxone from 13.49 DDD/100BD to $14.41 \mathrm{DDD} / 100 \mathrm{BD}$ and cefepime from 0.60 to 0.68 DDD/100BD in the first quarter of 2018 vs. same period of 2019, respectively). All the above-mentioned resulted
Table 2: Antibiotic consumption in the first quarters of 2018 and 2019 at the University Clinic for Pediatric Diseases in Skopje

\begin{tabular}{|c|c|c|c|}
\hline ATC & Form & Q1/2018 & Q1/2019 \\
\hline J01CA01 & Parenteral & 0.89 & 0.97 \\
\hline J01CA04 & Oral & 0.45 & 0.09 \\
\hline JOICA & & 1.33 & 1.07 \\
\hline J01CE10 & Oral & 0.15 & 0.05 \\
\hline J01CF02 & Oral & 0.88 & 0.51 \\
\hline J01CR02 & Oral & 0.82 & 0.42 \\
\hline J01CR05 & Parenteral & 0.26 & 0.23 \\
\hline JOIC & & 3.44 & 2.27 \\
\hline J01DB05 & Oral & 0.28 & 0.16 \\
\hline J01DB & & 0.28 & 0.16 \\
\hline J01DC02 & Parenteral & 0.00 & 0.01 \\
\hline J01DC04 & Oral & 0.18 & 0.09 \\
\hline J01DC & & 0.18 & 0.10 \\
\hline J01DD01 & Parenteral & 0.18 & 2.15 \\
\hline J01DD02 & Parenteral & 1.18 & 1.02 \\
\hline J01DD04 & Parenteral & 13.49 & 14.41 \\
\hline J01DD08 & Oral & 1.06 & 0.73 \\
\hline J01DD & & 15.90 & 17.58 \\
\hline J01DE01 & Parenteral & 0.60 & 0.68 \\
\hline J01DE & & 0.60 & 0.68 \\
\hline J01D & & 16.96 & 18.52 \\
\hline J01DH02 & Parenteral & 1.88 & 2.71 \\
\hline J01DH51 & Parenteral & 1.56 & 0.30 \\
\hline J01DH & & 3.44 & 3.01 \\
\hline J01EE01 & Oral & 0.00 & 0.59 \\
\hline J01E & & 0.00 & 0.59 \\
\hline J01FA03 & Oral & 0.00 & 0.11 \\
\hline J01FA10 & Oral & 1.97 & 0.81 \\
\hline J01FA & & 1.97 & 0.92 \\
\hline J01FF01 & Parenteral & 0.06 & 0.06 \\
\hline J01FF02 & Parenteral & 0.29 & 0.00 \\
\hline J01FF & & 0.35 & 0.06 \\
\hline J01GB03 & Parenteral & 0.13 & 0.18 \\
\hline J01GB06 & Parenteral & 3.21 & 3.50 \\
\hline J01G & & 3.35 & 3.68 \\
\hline J01MA02 & Parenteral & 0.20 & 0.24 \\
\hline J01MA02 & Oral & 1.79 & 0.22 \\
\hline J01MA & & 1.98 & 0.46 \\
\hline J01MB04 & Oral & 0.00 & 0.18 \\
\hline J01M & & 1.98 & 0.64 \\
\hline J01XA01 & Parenteral & 1.57 & 0.79 \\
\hline J01XA02 & Parenteral & 0.00 & 0.07 \\
\hline J01XA & & 1.57 & 0.86 \\
\hline J01XB01 & Parenteral & 0.59 & 0.73 \\
\hline J01XB & & 0.59 & 0.73 \\
\hline J01XD01 & Oral & 0.00 & 0.04 \\
\hline J01XX08 & Parenteral & 0.00 & 0.05 \\
\hline J01XX & & 2.15 & 1.68 \\
\hline TOTAL & & 33.65 & 31.37 \\
\hline
\end{tabular}

in a total increase of cephalosporin consumption from 16.96 to $19.25 \mathrm{DDD} / 100 \mathrm{BD}$ in the same period in $20181^{\text {nd }} 2019$ in the clinic. Declination in the group of carbapenems consumption was noticed (3.44 DDD/100BD in 2018 vs. 3.01 DDD/100BD in 2019), as a result of significant declination of combination imipenem, cilastatin from 1.56 to $0.30 \mathrm{DDD} / 100 \mathrm{BD}$, although the consumption of meropenem increased for $0.83 \mathrm{DDD} / 100 \mathrm{BD}$ in the evaluated period. Evident declination of penicillin's consumption was established in evaluated quarters in the year 2018 versus 2019, from 3.44 to $2.27 \mathrm{DDD} / 100 \mathrm{BD}$, respectively. Decrease of penicillin consumption was result of significant declination of broad-spectrum penicillin's (amoxicillin), beta-lactamase sensitive (benzathine phenoxymethylpenicillin), and combined penicillin's with beta-lactamase inhibitors (amoxicillin and clavulanic acid) consumption. Regarding macrolides consumption, midecamycin was introduced in 2019 as a therapeutic option searing $0.11 \mathrm{DDD} / 100 \mathrm{BD}$ in the first quarter of 2019 and declination of azithromycin for more than 1 DDD/100BD (1.97 in 2018 vs., 0.81 DDD/100BD in 2019) resulting in an overall decrease of macrolides consumption from 1.97 to 0.92 DDD/100BD in 2019. The therapeutic use of clindamycin remains the same 
in the first quarter of the year 2018 and 2019, 0.06 DDD/100BD, but important declination of lincomycin use was noticed and this antibiotic is not utilized in 2019. Concerning aminoglycosides, there was a small increase from 3.35 DDD/100BD in 2018 versus 3.68 DDD/100BD in 2019, with comparable consumption of gentamicin and amikacin in both years. Significant declination of quinolone consumption was confirmed in the evaluated period from 1.98 DDD/100BD to 0.64 DDD/100BD in the first quarter of 2018 and 2019, respectively, which could be result of most recent findings and recommendations regarding restrictions of quinolone indication field and limited prescription of this antibiotic group addressed by European Medicinal Agency (EMA) and Food and Drug Administration (FDA). From the group of glycopeptide

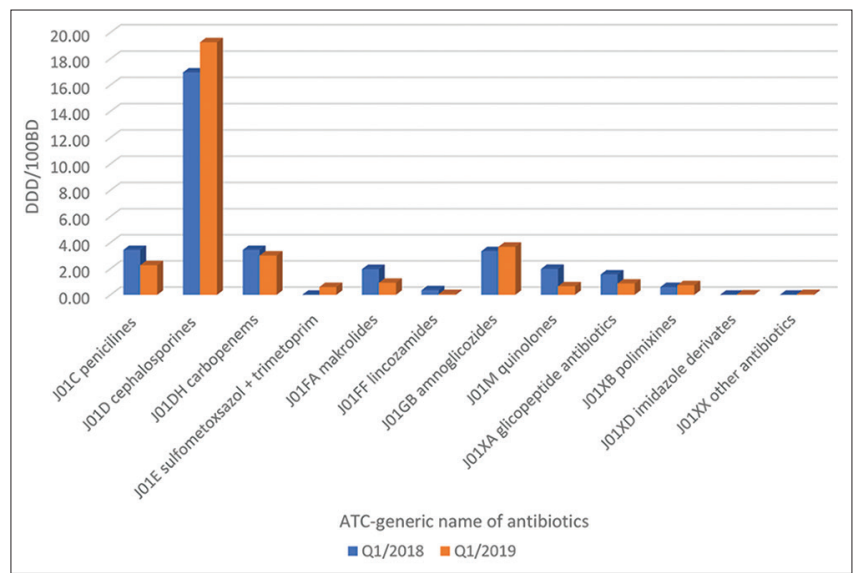

Figure 1: Comparison of antibiotics consumption in the University Clinic for Pediatric Diseases in Skopje in the first quarter of 2018 versus the first quarter of 2019

antibiotics, serious declination of vancomycin consumption was confirmed from 1.57 DDD/100BD 2018 to $0.79 \mathrm{DDD} / 100 \mathrm{BD}$ in 2019. Teicoplanin was introduced in 2019 and it reached a share of 0.07 DDD/100BD of antibiotics consumption in the first trimester in this year. The consumption of polymyxins (colistin) increased from 0.59 to 0.73 DDD/100BD, as well as consumption of imidazole derivatives (metronidazole for 0.04 DDD/100BD) and linezolid for 0.05 DDD/100BD (data presented in Figure 1).

The results show that the consumption of cephalosporins was $50.39 \%$ in the first quarter of 2018, rising up to $59.99 \%$ in the first quarter of 2019. Widening in the group of sulfonamides (up to 1.84\%) and aminoglycosides (from $9.95 \%$ to $11.45 \%$ ) consumption was confirmed in the evaluated first trimesters in the year 2018 versus 2019. Important decrease in dispensing share was confirmed for the group of carbapenems from $10.23 \%$ in 2018 to $9.38 \%$ in 2019 , for penicillins from $10.24 \%$ to $7.06 \%$ in 2018 compared to the same trimester in 2019 , for the group of quinolones from $5.9 \%$ to $2.01 \%$, and same declination for glycopeptide antibiotics from $4.66 \%$ to $2.68 \%$. In addition, decrease in consumption was proofed for the macrolides from 5.86 to $2.86 \%$ in the same evaluated period (data presented in Figure 2).

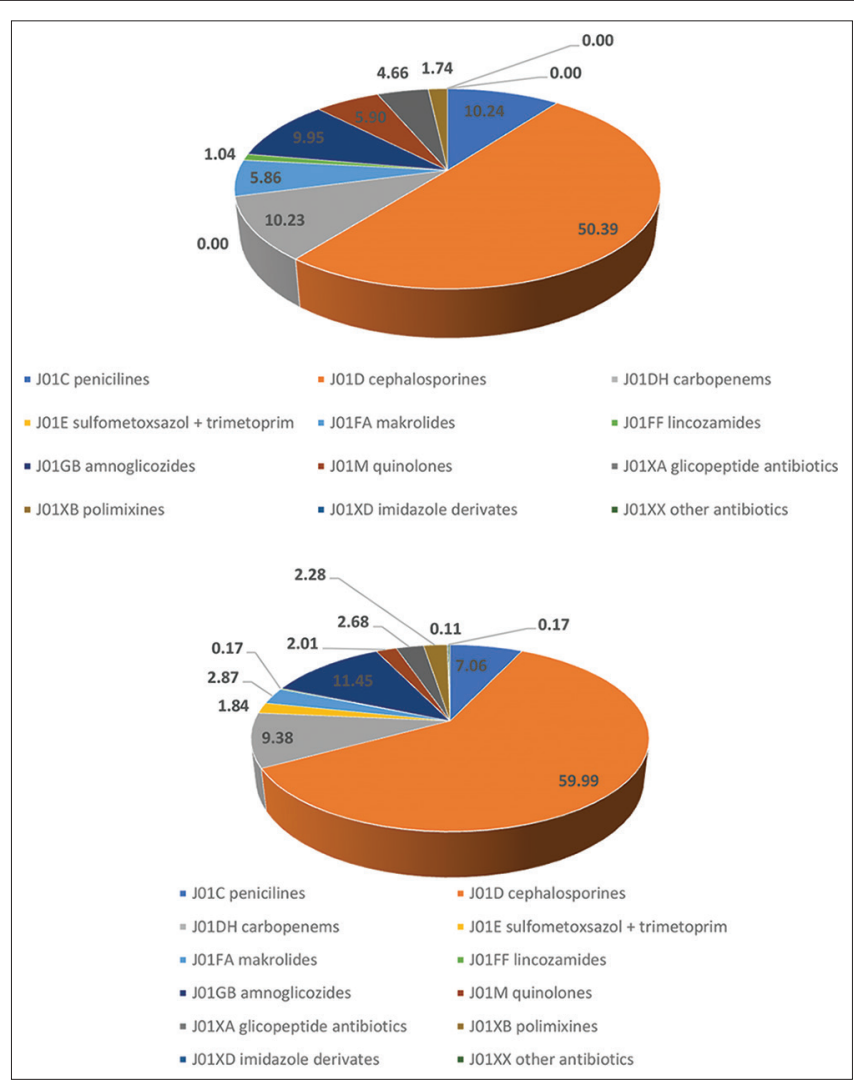

Figure 2: Percentage dispensing share of antibiotics in the University Clinic for Pediatric Diseases in Skopje in the first quarters of 2018 versus 2019

\section{Discussion}

Republic of North Macedonia has recently witnessed a nationwide outbreak of measles. In the first quarter of 2019 at the University Clinic for pediatric diseases, 138 children were hospitalized with respiratory complications (bronchopneumonia) induced by measles infection. Pneumonia is the most common severe complication of measles and is treated with ceftriaxone, while more complicated cases were treated with additional vancomycin and azithromycin. It should be considered that this fact has an impact on the results on antibiotic consumption in 2019.

It is intuitive that antibiotic resistance is predominantly confirmed in environments with high antibiotic consumption [17], [18], [19]. The overall usage and use of different classes of antibiotics in hospitals in Europe vary considerably. Results from 30 European countries regarding antibacterials consumption for systemic use (ATC group J01) that included 139 hospitals have confirmed that the median use was 49.6 DDD/100BD, whereas, in Southeast Europe, the median use was $42.3 \mathrm{DDD} / 100 \mathrm{BD}$. The encouraging fact is that the results from this study are lower than the median values in Europe confirmed in 2001, which indicates the improvement of rational antibiotic consumption [20].

Only a few studies were conducted for antibiotic consumption in hospitals in the Balkan region 
in recent years. The results from the study conducted at the Pediatric Clinic in Novi Sad in 2010 confirmed that antibiotic consumption was 35.48 DDD/100BD, with the highest share of a class of parenteral cephalosporin consumption with 9.89 DDD/100BD and ceftriaxone 4.88 DDD/100IBD [21]. The same trend was established for the parenteral cephalosporin consumption with 14.84 DDD/100BD in 2018, rising up to 17.58 DDD/100BD in 2019 at the University Clinic for pediatric diseases.

In the year 2018, we have confirmed that penicillin's and carbapenems consumption are second in consumption share in the University Clinic for pediatric diseases with 3.44 DDD/100BD in 2018 decreased to $2.27 \mathrm{DDD} / 100 \mathrm{BD}$ in 2019 followed by the group of aminoglycosides with 3.35 DDD/100BD in 2018 and approximately same values of 3.68 DDD/100BD in 2019. The group of macrolides has the same consumption in both years of 1.97 DDD/100BD. Quinolones have consumption of 0.68 in 2018 and 0.92 DDD/100BD in 2019 , respectively. Our data are comparable with data on antibiotic consumption in Republic of Croatia (23).

The results obtained through an organization called Iskra that collects and processes data for antibiotics consumption in the Republic of Croatia confirmed that annual consumption of antibiotic consumption in the Republic of Croatia in the period from 2010 to 2017 is $42.34 \pm 1.7$ DDD/100BD [22]. The most frequently dispensed antibiotics are the group of cephalosporins $13.23 \pm 0.75 \mathrm{DDD} / 100 \mathrm{BD}$, followed by the group of penicillin with an average of $12.34 \pm 0.47$ DDD/100BD, macrolides3.17 \pm 0.15 DDD/100BD, and aminoglycosides $2.41 \pm 0.26 \mathrm{DDD} / 100 \mathrm{BD}$, the same pattern as in the pediatric clinic in the Republic of North Macedonia in the year 2019.

Data on hospital antibiotic consumption in Croatia for 2017 ranges from 26.5 to 139.5 DDD/100BD, average 45,30 DDD/100BD. In comparison, the results from the University Clinic for pediatric diseases show reduced consumption of the antibiotics, while the same class of antibiotics - cephalosporins of the third generation - is the most frequently dispensed antibiotics in both studies.

Most recent relevant results obtained from the hospital in Canton Sarajevo in Bosnia and Hercegovina revealed that antimicrobial consumption in 2016 was 41.13 DDD/100BD and has raised up to 45.55 DDD/100BD in 2017. In both years, the group of cephalosporins was the most frequently dispensed antibiotics, but the first generation of cephalosporins has the highest consumption, which is the main difference to the results from The University Clinic for Pediatric Diseases where the third generation of cephalosporins was on the first place for consumption [23].

The results from the European Surveillance of Antimicrobial Consumption Network (ESAC-Net) in the period from 2000 to 2014 confirmed that antibiotic consumption has mostly increased in England,
Belgium, Ireland, and Greece and that consumption trends were not permanent and they change during the evaluated period [24]. The differences in the extent of antibiotics consumption among the countries depend on different antibiotic consumption policies, differences in prescription habits, patient population, antibiotic resistance, as well as measures applied for evaluation of antibiotics consumption. Mechanisms for rational antibiotics consumption are applied worldwide as a restrictive approach with a limited list of antibiotics as well as guidance for the physicians for rational prescription and administration [25]. Recognizing the factors that influence drug prescription habits and conducting studies to monitor bacterial resistance are one way to obtain relevant data and provide adequate information on the effectiveness of antibiotics, which allows prescribing the most appropriate therapy.

\section{Conclusion}

The benefits of monitoring the antibiotic prescribed pattern are critical due to the fact that they provide adequate data on consumption of antibiotics and adherence to guidelines. Reduction in total antibiotic consumption with ATC code J01 for the first quarter (3 months - January, February, and March) in the year 2019 of 32.09 DDD/100BD was confirmed compared to the same period in 2018 at the University Clinic for Pediatric Diseases in Skopje is 33.65 DDD/100BD. The most consummated antibiotics in the $1^{\text {st }}$ trimester of both years were the group of cephalosporins, especially the $3^{\text {rd }}$ - and $4^{\text {th }}$-generation parenteral cephalosporins with from 16.96 to $19.25 \mathrm{DDD} / 100 \mathrm{BD}$ in the evaluated period due to measles outbreak. Decrease of penicillin's, carbapenems, macrolides, and quinolones was confirmed in the analyzed period. The most commonly used drugs remain ceftriaxone followed by amikacin in 2019 but azithromycin administrated orally significantly declined. The third most commonly utilized antibiotic in first quarter of 2019 become meropenem, antimicrobial drug from the group of carbapenems.

\section{References}

1. EASAC (Council). Tackling Antibacterial Resistance in Europe. Available from: https://www.easac.eu/fileadmin/PDF_s/reports statements/Tackling.pdf. [Last accessed on 2019 November 10].

2. European Centre for Disease Prevention and Control. Antimicrobial Consumption. In: Annual Epidemiological Report for 2017 Antimicrobial Consumption. Sweden: European Centre for Disease Prevention and Control;

3. Available from: https://www.ecdc.europa.eu/sites/portal/files/ documents/AER_for_2017-antimicrobial-consumption.pdf. 
[Last accessed on 2019 November 10].

4. Republic of North Macedonia. National Strategy for Antimicrobial Resistance AMR in the Republic of North Macedonia 20192023. Republic of North Macedonia; 2019.

5. Antimicrobial Resistance. Available from: http://www.ecdc. europa.eu/en/healthtopics/antimicrobial_resistance/Pages/ index.aspx. [Last accessed on 2019 November 10].

6. Antimicrobial Use. Available from: http://www.who.int/ drugresistance/use/en. [Last accessed on 2019 November 10].

7. Central Asian and Eastern European Surveillance of Antimicrobial Resistance. Annual Report 2016. Available from: http://www.euro.who.int/_data/assets/pdf_file/0006/285405/ CAESAR-surveillance-antimicrobial-resistance2016.pdf. [Last accessed on 2019 November 10]

8. Chaw PS, Schlinkmann KM, Raupach-Rosin $H$, Karch $A$, Pletz MW, Huebner $J$, et al. Antibiotic use on paediatric inpatientsin a teaching hospital in the Gambia,a retrospective study. Antimicrob Resist Infect Control. 2018;7:82.

PMid:30026940

9. Sharma R, Sharma CL, Kapoor B. Antibacterial resistance: Current problems and possible solutions. Indian J Med Sci. 2005;59(3):120-9. https://doi.org/10.4103/0019-5359.15091 PMid:15805685

10. Al-Tawfiq JA. Antibiotic resistance of pediatric isolates of Streptococcus pneumoniae in a Saudi Arabian hospital from 1999 to 2004. Med Sci Monit. 2006;12(11):CR471-5. PMid:17072272

11. Bartoloni A, Pallecchi L, Benedetti M, Fernandez C, Vallejos Y, Guzman E, et al. Multidrug-resistant commensal Escherichia coli in children, Peru and Bolivia. Emerg Infect Dis. 2006;12(6):90713. https://doi.org/10.3201/eid1206.051258

12. Fritzsche M, Ammann RA, Droz S, Bianchetti MG, Aebi C. Changes in antimicrobial resistance of Escherichia coli causing urinary tract infections in hospitalized children. Eur J Clin Microbiol Infect Dis. 2005;24(3):233-5. https://doi.org/10.1007/ s10096-005-1301-2 PMid:15772820

13. Al-Tawfiq JA, Anani AA. Antimicrobial susceptibility pattern of bacterial pathogens causing urinary tract infections in a Saudi Arabian hospital. Chemotherapy. 2009;55(2):127-31. https://doi. org/10.1159/000198698

PMid:19188712

14. von Gunten V, Troillet N, Beney J, Boubaker K, Lüthi JC, Taffé $\mathrm{P}$, et al. Impact of an interdisciplinary strategy on antibiotic use: A prospective controlled study in three hospitals. J Antimicrob Chemother. 2005;55(3):362-6. https:// doi.org/10.1093/jac/dki021

PMid:15705638

15. Polk RE, Fox C, Mahoney A, Letcavage J, MacDougall C. Measurement of adult antibacterial drug use in 130 US hospitals: Comparison of defined daily dose and days of therapy. Clin Infect Dis. 2007;44(5):664-70. https://doi.org/10.1086/511640

\section{PMid:17278056}

16. World Health Organization. Collaborating Centre for Drug Statistics Methodology, Guidelines for ATC classification and DDD Assignment 2018. Oslo, Norway: World Health Organization; 2017.

17. Rogues AM, Dumartin C, Amadéo B, Venier AG, Marty N Parneix $\mathrm{P}$, et al. Relationship between rates of antimicrobial consumption and the incidence of antimicrobial resistance in Staphylococcus aureus and Pseudomonas aeruginosa isolates from 47 French hospitals. Infect Control Hosp Epidemiol. 2007;28(12):1389-95. https://doi.org/10.1086/523280 PMid:17994520

18. Plüss-Suard C, Pannatier A, Kronenberg A, Mühlemann K, Zanetti G. Impact of antibiotic use on carbapenem resistance in Pseudomonas aeruginosa: Is there a role for antibiotic diversity? Antimicrob Agents Chemother. 2013;57:1709-13. https://doi. org/10.1128/aac.01348-12 PMid:23357763

19. Gbaguidi-Haore $H$, Dumartin $C$, L'Hériteau $F$, Péfau $M$, Hocquet D, Rogues AM, et al. Antibiotics involved in the occurrence of antibiotic-resistant bacteria: A nationwide multilevel study suggests differences within antibiotic classes. J Antimicrob Chemother. 2013;68(2):461-70. https:// doi.org/10.1093/jac/dks406 PMid:23075690

20. Available from: http://www.whocc.no/filearchive/ publications/1_2013guidelines.pdf. [Last accessed on 2019 November 10].

21. Jakovljevic E, Ilić K, Jelesić Z, Konstantinidis G. A one-year prospective study on the antibiotic resistance of E-coli strains isolated in urinary specimens of children hospitalized at the University Pediatric Medical Center in Novi Sad, Serbia. Infection. 2013;41(6):1111-9. https://doi.org/10.1007/s15010-013-0493-0

22. ISKRA, Antimicrobial Resistance Surveillance in Human Medicine CROATIA, Pracenje Otpornosti Bakterija na Antibiotike HRVATSKA. Available from: http://www.iskra.bfm.hr/hrv/Default. aspx. [Last accessed on 2019 November 10].

23. Review of Antibiotics Expenditure in the Canton Sarajevo in Period 2016/2017. Available from: https://www.mz.ks.gov. ba/sites/mz.ks.gov.ba/files/izvjestaj_o_potrosnji_antibiotika za_2016_2017_parafirano.pdf. [Last accessed on $201 \overline{9}$ November 10].

24. Gould IM, Lawes T. Antibiotic stewardship: Prescribing socia norms. Lancet. 2016;387(10029):1699-701. https://doi. org/10.1016/s0140-6736(16)00007-6

PMid:26898851

25. Buising KL, Thursky KA, Robertson MB, Black JF, Street AC, Richards $\mathrm{MJ}$, et al. Electronic antibiotic stewardship reduced consumption of broad-spectrum antibiotics using a computerized antimicrobial approval system in a hospital setting. J Antimicrob Chemother. 2008;62(3):608-16. https://doi.org/10.1093/jac/ dkn218

PMid:18550680 